Chronic myelogenous leukemia

\title{
Chronic myeloid leukemia management at the time of the COVID-19 pandemic in Italy. A campus CML survey
}

\author{
Massimo Breccia' - Elisabetta Abruzzese $\mathbb{1}^{2}{ }^{2}$ - Monica Bocchia ${ }^{3}$ - Massimiliano Bonifacio $\oplus^{4}$ - Fausto Castagnetti ${ }^{5}$. \\ Carmen Fava $\mathbb{D}^{6}$. Sara Galimberti $\mathbb{D}^{7}$ - Antonella Gozzini ${ }^{8}$ - Gabriele Gugliotta ${ }^{5}$ - Alessandra lurlo ${ }^{9}$. \\ Roberto Latagliata $^{1}$ - Luigiana Luciano ${ }^{10}$ - Patrizia Pregno ${ }^{11}$ - Giovanna Rege-Cambrin ${ }^{12}$ - Gianantonio Rosti ${ }^{5}$

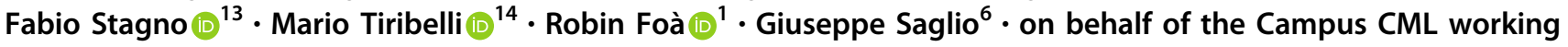 \\ group
}

Received: 9 May 2020 / Revised: 28 May 2020 / Accepted: 1 June 2020 / Published online: 18 June 2020

c) Springer Nature Limited 2020

It has been reported that imatinib may have a specific protective role in blocking the fusion of the protein $S$ of the Coronavirus belonging to the viral surface with cell membranes [1]. This role could prevent the endocytosis necessary for the viral activation of different viral species (Sars-COV, MERS-CoV, and IBV) [2]. No in vivo information has been reported so far. The only information related to chronic

Members of the Campus CML working group are listed below Acknowledgements.

Robin Foà

rfoa@bce.uniroma1.it

1 Department of Precision and Translational Medicine, Sapienza University, Rome, Italy

2 Hematology, Sant'Eugenio Hospital, Rome, Italy

3 UOC Hematology, University of Siena, Siena, Italy

4 Hematology, Policlinico GB Rossi, University of Verona, Verona, Italy

5 Department of Hematology/Oncology, University of Bologna, Bologna, Italy

6 Azienda Ospedaliera Mauriziano, Torino, Italy

7 University of Pisa, Pisa, Italy

8 Ospedale Careggi, Firenze, Italy

9 Fondazione IRCSS Ca' Granda Ospedale Maggiore Policlinico of Milan, Milan, Italy

10 Federico II University, Naples, Italy

11 Ospedale Città della Salute e della Scienza, Turin, Italy

12 Ospedale San Luigi, Orbassano, Italy

13 Ospedale Ferrarotto-University of Catania, Catania, Italy

14 University of Udine, Udine, Italy myeloid leukemia (CML) comes from a relatively small series from the Hubei Province where it was found that relatively few COVID-19+ CML patients were reported [3].

The Campus CML is an ongoing program aimed at creating a network of physicians in Italy involved in different aspects of the management of patients with CML, sharing experiences and updates for the diagnosis, treatment of the disease, identification and prevention of the specific toxicity of the drugs used but also on possible future therapeutic approaches. Considering the current COVID-19 emergency in Italy, we asked Italian clinicians about the incidence of infections among their CML patients and about the management of the disease in this unique pandemic period. On April 6 we sent an ad hoc questionnaire to 51 Campus CML centers throughout the country and 47 centers $(92 \%)$ have completed the online survey. Nineteen centers were from the most affected regions: Lombardia, Piemonte, Veneto, Emilia-Romagna. Twelve centers (42.5\%) reported a decrease in the incidence of new CML cases in this period. Data from a large cohort of 6883 CML patients were gathered: only 12 cases of confirmed COVID-19 infection were reported $(0.17 \%)$ up to the middle of April. Two of the 12 cases were healthcare professionals and 8 have been infected in the most affected Italian regions. Only two deaths have been recorded, one in a patient aged 91 years. Other five patients were suspected based on the symptoms presented, but tested negative. The majority of participants $(89 \%)$ declared that CML patients were tested only in case of fever and/or related symptoms and/or who had been in close contact with a positive subject, but not routinely. In Italy, testing is routinely performed on inpatients. Eight centers (17\%) reported difficulties in performing a baseline diagnostic work-up and molecular monitoring to detect MRD during treatment. Sixty-six percent of centers postponed the molecular analysis by 1-2 months during this emergency if 
patients were at least in MR3 or if they were in deep and stable molecular response. Patients who did not have to perform a molecular monitoring were checked prevalently by telephone or by email during the COVID-19 pandemic. The COVID-19 emergency had a repercussion on the treatmentfree remission (TFR) strategy: $58 \%$ of participating physicians did not propose a possible discontinuation and $24 \%$ of patients already in TFR have had to modify the molecular monthly monitoring approach. We recorded changes also in the delivery of drugs: while imatinib delivery was not affected due to local pharmacy distributions, $36 \%$ of physicians reported consequences for second-generation TKIs subject to AIFA (the Italian Medicine Agency) monitoring in Italy. In some instances, delivery was carried out directly at the patient's home (12\%) or a supply for more months has been granted $(10 \%)$ by the treating center.

The activity of ongoing CML trials has already been affected by the emergency: $34 \%$ of physicians have stopped enrollment and $8 \%$ have continued with the ongoing trials with some difficulties in the planned follow-up of enrolled patients. AIFA issued a decree that allowed patients enrolled in a trial and not able to reach the center to perform the requested tests at a hospital near home with a complete reimbursement: $51 \%$ of interviewed centers adopted this strategy.

These results of our survey show that the incidence of COVID-19 infection has so far proven extremely low in CML patients treated with TKIs. These data are in line with what observed in adult patients with $\mathrm{Ph}+$ acute lymphoblastic leukemia $(\mathrm{Ph}+\mathrm{ALL})$ in Italy, where patients are induced with a TKI plus steroids and no systemic chemotherapy, and could continue to be managed even at the peak of the COVID-19 outbreak [4]. Taken together, the data gathered on over 7000 cases of CML and Ph+ ALL support a potential role of TKIs in protecting patients from COVID-19 infection. To conclusively answer this question a randomized study (EudraCT 2020-001236-10) is verifying the effect of imatinib in preventing pulmonary vascular leak in patients with severe COVID-19 disease. Although the likelihood of developing a symptomatic COVID-19 infection in CML patients in Italy is close to zero and lower than that of the general population in Italy, the current pandemic emergency is, however, already negatively impacting on different aspects of the day-to-day management of patients, on disease monitoring and on treatment decisions, as well as on the enrollment in and compliance to clinical trials.

Acknowledgements The authors wish to thank all the Campus CML colleagues who completed the survey.

Campus CML working group Miggiano Maria Cristina ${ }^{15}$, Capodanno Isabella $^{16}$, Accurso Vincenzo ${ }^{17}$, Sorà Federica ${ }^{18}$, Luzi Debora ${ }^{19}$,

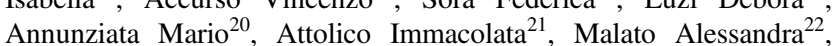
Sancetta Rosaria ${ }^{23}$, Elena Chiara ${ }^{24}$, Barulli Sara ${ }^{25}$, Scortechini Anna
Rita $^{26}$, Leonetti-Crescenzi Sabrina ${ }^{27}$, Tafuri Agostino ${ }^{28}$, Cavazzini Francesco $^{29}$, Caocci Giovanni ${ }^{30}$, Lucchesi Alessandro ${ }^{31}$, Rapezzi Davide $^{32}$, Pizzuti Michele ${ }^{33}$, Binotto Gianni ${ }^{34}$, Intermesoli Tamara ${ }^{35}$, Maggi Alessandro ${ }^{36}$, Crisa' Elena ${ }^{37}$, Crugnola Monica ${ }^{38}$, D' Adda Mariella $^{39}$, Beltrami Germana ${ }^{40}$, Lunghi Francesca ${ }^{41}$, Vincelli Iolanda -Donatella ${ }^{42}$, Sanpaolo Grazia ${ }^{43}$, Franceschini Luca ${ }^{44}$, Russo Sabina ${ }^{45}$, La Barba Gaetano ${ }^{46}$, Levato Luciano ${ }^{47}$

${ }^{15}$ U.O.C. Ematologia, Vicenza, Italy; ${ }^{16} \mathrm{Az}$. Unità Sanitaria IRCCS Reggio-Emilia, Reggio-Emilia, Italy; ${ }^{17}$ UOC Ematologia Policlinico Paolo Giaccone Palermo, Palermo, Italy; ${ }^{18}$ Università CattolicaPoliclinico A. Gemelli, IRCSS, Roma, Italy; ${ }^{19}$ OncoematologiaAzienda Ospedaliera Santa Maria, Terni, Italy; ${ }^{20} \mathrm{Az}$. Ospedaliera Cardarelli, Napoli, Italy; ${ }^{21}$ Ematologia con Trapianto Policlinico Bari, Bari, Italy; ${ }^{22}$ UOC Ematologia ad indirizzo oncologico Ospedali Riuniti Villa Sofia-Cervello, Palermo, Italy; ${ }^{23}$ UO Ematologia OC dell'Angelo Mestre-Venezia, Venezia- Mestre, Italy; ${ }^{24}$ Fondazione IRCCS Policlinico San Matteo, Pavia, Italy; ${ }^{25}$ U.O.C. Ematologia, Pesaro, Italy; ${ }^{26}$ Clinica Ematologia, Ancona, Italy; ${ }^{27}$ azienda ospedaliera San Giovanni-Addolorata, Roma, Italy; ${ }^{28} \mathrm{~S}$. Andrea - Sapienza -Azienda Ospedaliera Universitaria, Roma, Italy; ${ }^{29} \mathrm{Az}$. OspedalieroUniversitaria S. Anna Ferrara, Ferrara, Italy; ${ }^{30}$ Ospedale Businco, Cagliari, Italy; ${ }^{31}$ Istituto Scientifico Romagnolo per lo Studio e la Cura dei Tumori (IRST) IRCCS, Meldola, Italy; ${ }^{32}$ S.C. Ematologia A.S.O. Santa Croce e Carle di Cuneo, Cuneo, Italy; ${ }^{33}$ Ematologia Ospedale San Carlo, Potenza, Italy; ${ }^{34}$ UOC Ematologia ed Immunologia Clinica- Università di Padova, Padova, Italy; ${ }^{35}$ Papa Giovanni XXIII, Bergamo, Italy; ${ }^{36}$ s.c. ematologia con trapianto, Taranto, Italy; ${ }^{37} \mathrm{AOU}$ Maggiore della Carità, Novara, Italy; ${ }^{38} \mathrm{AOU}$ Parma, Parma, Italy; ${ }^{39} \mathrm{U}$. O. Ematologia, Brescia, Italy; ${ }^{40}$ U.O Ematologia Ospedale Policlinico S. Martino, Genova, Italy; ${ }^{41}$ Ospedale San Raffaele MI, Milano, Italy; 42divisione di ematologia ospedale Bianchi -Melacrino-Morelli, Reggio-Calabria, Italy; ${ }^{43}$ Ematologia Casa Sollievo della Sofferenza, San Giovanni Rotondo, Italy; ${ }^{44}$ Policlinico Tor Vergata, Roma, Italy; 45AOU G. Martino Policlinico di Messina, Messina, Italy; ${ }^{46}$ Ematologia Clinica Ospedale Civile "Spirito Santo", Pescara, Italy; ${ }^{47}$ Ematologia Catanzaro, Catanzaro, Italy

\section{Compliance with ethical standards}

Conflict of interest The authors declare that they have no conflict of interest.

Publisher's note Springer Nature remains neutral with regard to jurisdictional claims in published maps and institutional affiliations.

\section{References}

1. Coleman CM, Sisk JM, Mingo RM, Nelson EA, White JM, Frieman MB. Abelson kinase inhibitors are potent inhibitors of severe acute respiratory syndrome coronavirus and middle east respiratory syndrome coronavirus fusion. J Virol. 2016;90:8924-8933.

2. Sisk JM, Frieman MB, Machamer CE. Coronavirus S proteininduced fusion is blocked prior to hemifusion by Abl kinase inhibitors. J Gen Virol. 2018;99:619-630.

3. Li W, Wang D, Guo J, Yuan G, Yang Z, Gale RP, et al. COVID-19 in persons with chronic myeloid leukemia. Leukemia. 2020:1-6. https://doi.org/10.1038/s41375-020-0853-6.

4. Foà R, Bonifacio M, Chiaretti S, Curti A, Candoni A, Fava C, et al. $\mathrm{Ph}+$ acute lymphoblastic leukaemia in italy during the covid-19 pandemic. A campus ALL study. Br J Haematol. 2020. https://doi. org/10.1111/bjh.16758. 